\title{
Recurrent intra-abdominal arterial bleedings after severe cough
}

Jee-min $\mathrm{Kim}^{1}$ and Deog Kyeom Kim²,3

${ }^{1}$ Division of Pulmonary and Critical Care Medicine, National Medical Center, Seoul; ${ }^{2}$ Division of Pulmonary and Critical Care Medicine, Department of Internal Medicine, Seoul Metropolitan Government Seoul National University Boramae Medical Center, Seoul; ${ }^{3}$ Department of Internal Medicine, Seoul National University College of Medicine, Seoul, Korea
Received: August 24, 2018 Revised : September 4, 2018 Accepted: September 6, 2018

\section{Correspondence to}

Deog Kyeom Kim, M.D.

Tel: $+82-2-870-2228$

Fax: $+82-2-830-2826$

E-mail:kimdkmd@gmail.com
A 70-year-old man with a chronic obstructive pulmonary disease with a frequent history of exacerbation presented to emergency room with progressive pain in his right lower abdominal quadrant which had developed after severe coughing. He had no history of trauma or carrying a weighty burden. About $5 \times 5 \mathrm{~cm}$-sized mass was palpated at the right lower abdominal quadrant.
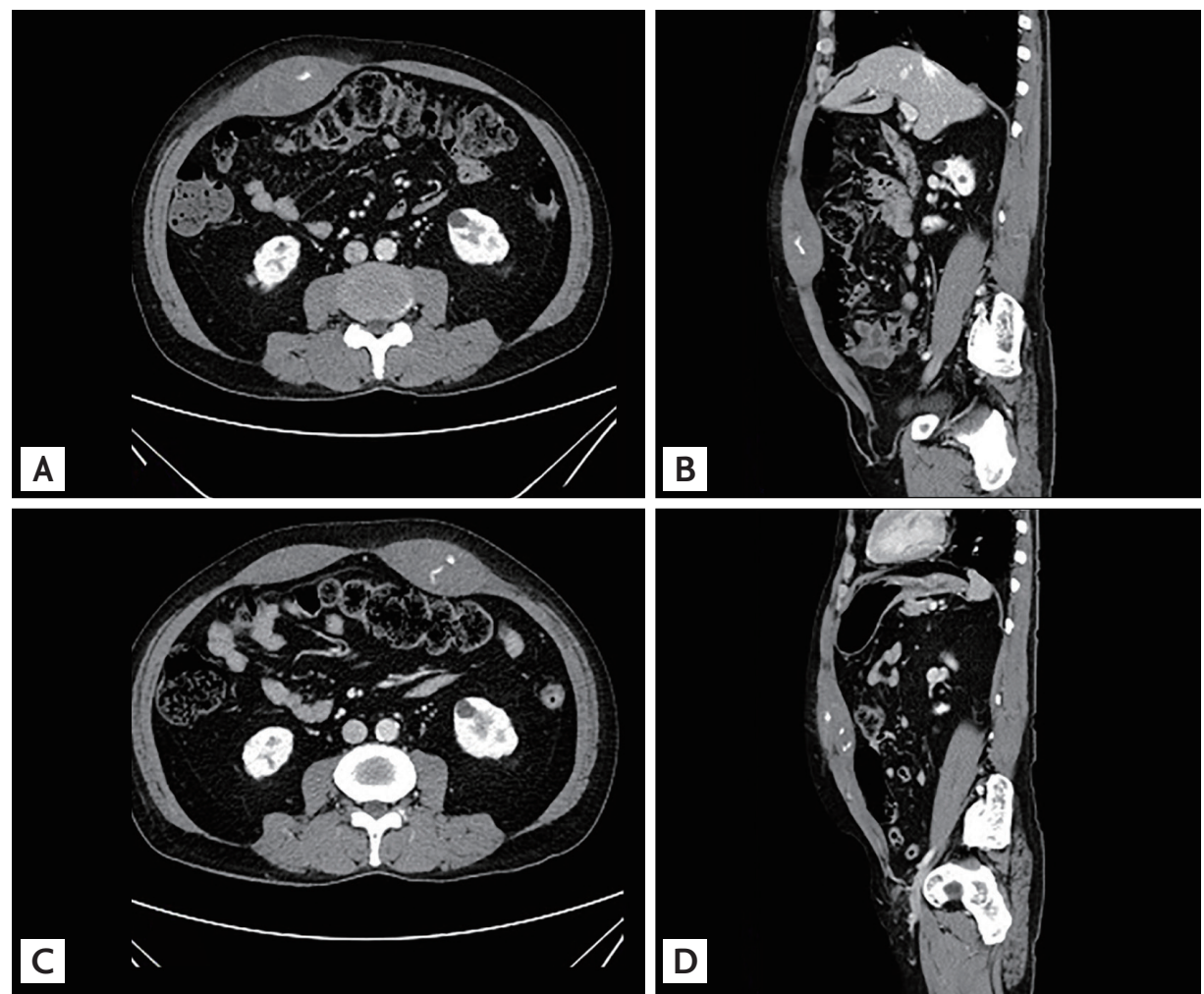

Figure 1. (A, B) Computed tomographic angiography at patient's arrival on emergency room revealed $6 \times 4 \mathrm{~cm}$ hematoma in right rectus abdominis muscle with active contrast extravasation. (C, D) Follow-up computed tomographic angiography revealed $7 \times 4 \mathrm{~cm}$ hematoma in left rectus abdominis muscle with contrast extravasation. Right side hematoma reduced in extent. 


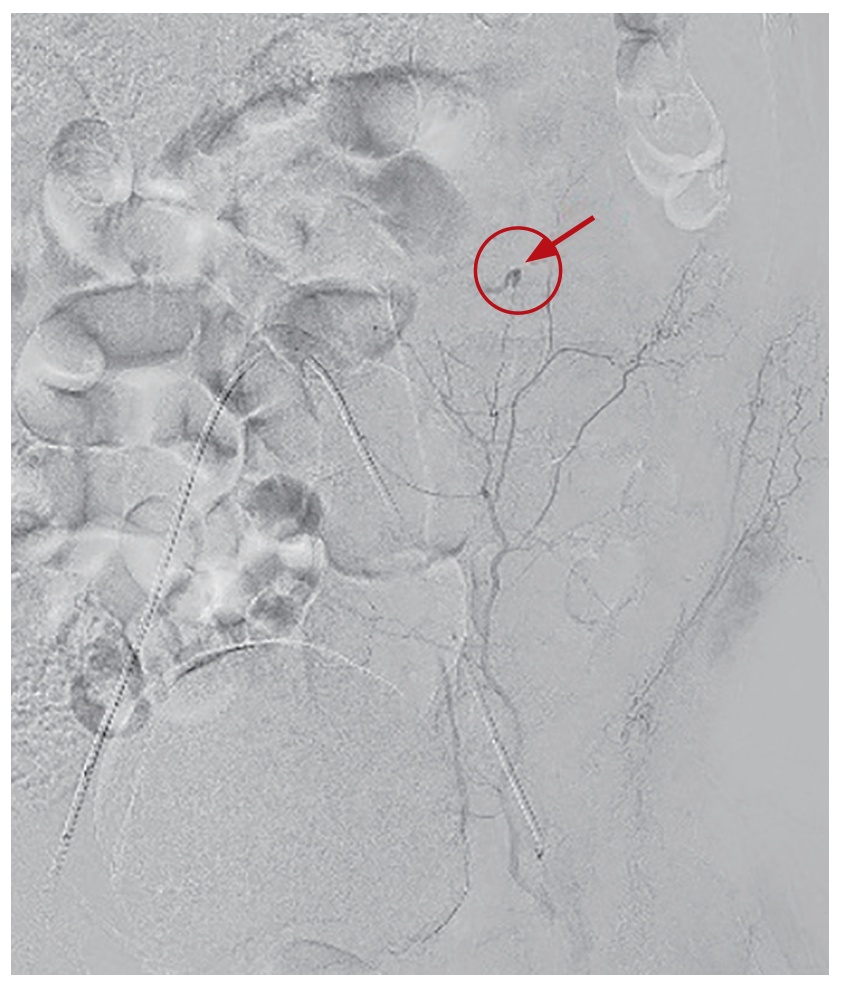

Figure 2. Angiography shows active contrast extravasation at left inferior epigastric artery (arrow and circle). Embolization was done with glue/lipiodol 1:3 mixture.

radiologic, laboratory, and physical examinations were performed but did not reveal abnormal results. Elevation of intra-abdominal pressure with severe coughing seemed responsible for the arterial bleeding in rectus muscles. Angiography with embolization of right internal mammary artery was performed. About a week later, he again complained of left lower quadrant abdominal pain with palpable mass after another severe coughing. While the CT angiography showed improving state of right intra-muscular hematoma, there was a newly developed hematoma with contrast extravasation in left rectus abdominal muscle (Fig. $1 \mathrm{C}$ and $1 \mathrm{D}$ ). Angiography with embolization was again performed. He was treated for another week with meticulous management for coughing, and was discharged after assuring that both hematomas were reducing in size with no evidence of further bleeding.

Severe cough is associated with several musculoskeletal complications, including rib fractures and abdominal wall herniation. Abdominal wall hematoma is an extremely rare complication of cough resulting from overstretching and overcontraction of the abdominal wall muscles. This case, as far as we know, is the first case of sequential bilateral hematoma that resulted from a cough that was successfully managed with embolization.

Written informed consent by the patients was waived due to a retrospective nature of our report.

\section{Conflict of interest}

No potential conflict of interest relevant to this article was reported. 